\title{
Determining amphibious command and control staffing requirements using business process modelling and simulation
}

\author{
$\underline{\text { S. Tavener }}^{\mathrm{a}}$ and P.M. Asenstorfer ${ }^{\mathrm{a}}$ \\ a Joint and Operations Analysis Division, Defence Science and Technology Group, Department of Defence \\ Email: sandra.tavener@dst.defence.gov.au
}

\begin{abstract}
The Australian Defence Organisation has recently significantly increased its amphibious capability. To support this, the associated Command and Control (C2) Headquarters (HQ), led by Commander Australian Amphibious Task Group (COMAUSATG), has grown by up to $600 \%$ in ten years. However, COMAUSATG has not had strong evidence that the size and composition of this staff were correct, and consequently requested DST conduct a study.

A business process simulation model had previously been built to provide initial staff numbers prior to the introduction of the new capability. The current study updated the model to reflect current amphibious command and control processes now that there was experience in using the amphibious capability. Data was collected from an exercise as well as via workshops. It was found that good quality data could be obtained from well facilitated workshops, but the data obtained when staff were asked to self-record their activities during an exercise was of lesser quality. To validate the data collected in workshops, direct observations of tasks performed during an exercise or operation is required. Additional work is also required to ensure the tasks described are a complete set of tasks that should be performed.
\end{abstract}

There were also limitations on which Business Process Model (BPM) techniques can be used to explore C2 organisations. BPM is suitable for use with $\mathrm{C} 2$ when determining the staff composition and exploring the work load of individual roles. However, the technique of adjusting processes to explore the efficiency improvements is very difficult because the processes are not well defined to begin with and the impacts of any changes are difficult to assess. As tasks are complex, they are rarely performed the same way and thus it is difficult to obtain task durations with any statistical robustness. Consequently, BPM is not particularly well suited to optimising the processes for a $\mathrm{C} 2$ organisation.

One component of the study was to understand the effect of different types of missions upon the HQ construct. Use-cases covering the spectrum of mission types were built to represent the type and rates of HQ tasks. From these use-cases, suitable probability variables used within the model were determined by subject matter experts. The content and detail contained within the use-cases shapes the subject matter expert's estimates of the variables; consequently, a use-case framework that leads to more consistency across experts is desired.

Presenting the final results in a form suitable for the end-user is a critical component of a well-designed study. For this study an interactive colour-coded organisational structure diagram allowed COMAUSATG to explore the results and provide their interpretation. Allowing COMAUSATG to define and change the thresholds of 'low' and 'high' tasking meant that different operational tempos and needs could be explored and its effects seen across their organisation.

Keywords: $\quad$ Workforce planning, business process modelling, defence, command and control, headquarters 
S Tavener and P.M. Asenstorfer, Determining amphibious command and control staffing requirements using business process modelling and simulation

\section{INTRODUCTION}

Since 2000 the Australian Government has articulated the need for a 'well-trained, well-equipped defence force that is available for operations at short notice, and one that can be sustained on deployment over extended periods' [Defence White paper (2000)]. This force will need to 'operate with decisive effect throughout the northern maritime and littoral approaches to Australia', 'be able to adapt to unfamiliar operating environments' and 'generate a wide range of capabilities which can be deployed for [such] tasks with very little warning' [Defence White paper (2009)]. To support this, the Australian Defence Organisation (ADO) has significantly increased its amphibious capability with the recent purchase of the amphibious capable ships, HMAS Canberra and HMAS Adelaide. As a consequence, the staff numbers for the ADO entity that provides the command and control (C2) for amphibious operations has grown in the order of $600 \%$ over the last ten years.

In 2016 the head of this entity, the Commander Australian Amphibious Task Group (COMAUSATG), was concerned this staff size may be incompatible with future Navy staffing priorities and COMAUSATG had no evidence, other than anecdotal, that the staffing numbers or composition were correct. To that end, COMAUSATG tasked the Defence Science and Technology (DST) Group to determine the actual amphibious C2 staffing requirements for different scales of amphibious operation. This information would be used to inform decisions and support arguments on the headquarters' (HQ) organisational design.

This paper describes the DST study undertaken. It covers the process modelling and simulation method selected, including any limitations. It discusses the variety of data collection methods that were used to update and extend the model, as well as the use-cases used in the study. It also provides examples of the process simulation model construct, describes how the simulation data was analysed, and provides an example of the spreadsheet tool provided to COMAUSATG.

\section{SIMULATION METHOD}

In 2009 DST performed a study to forecast the number and composition of staff required for a fully capable amphibious C2 HQ. At the start of the study a review was conducted on techniques being used within DST for workforce studies. Based on this review it was decided that business process modelling (BPM) would provide the level of detail required for designing a new amphibious C2 staff structure. So, a BPM simulation was developed from information available from Australian Defence Force (ADF) and North Atlantic Treaty Organisation doctrine. In 2016, due to the tight timeline COMAUSATG had for updated information, the decision was taken to update the 2009 model to reflect actual current practices.

BPM simulation involves modelling the sequence of tasks performed, as well as the resources (including personnel), information flow, and time required to perform the tasks. Outputs from such models include the time taken to complete a series of tasks and information on the time personnel resources are involved in tasks. By adjusting the model, users can investigate the impact on the model outputs when changing the tasks, sequence or allocation of resources. This is very useful when designing staff structures.

The main limitation of BPM is the level of detail required about the processes performed. Processes are a series of tasks performed to achieve an end goal. They can contain decision branches, loops and stop events. If the process is well defined and static, such as a factory floor building cars, it is relatively easy to model because the tasks are repeated. Collecting data over time will provide statistical robustness. It is significantly more difficult to model a system when the process steps 'depend'; when the number of decision branches is large and there are significant overlap between branches. This results in tasks not being repeated and prevents the collection of sufficient data to provide statistical robustness. This is the case for $\mathrm{C} 2$.

Despite this difficulty, valuable information can be obtained using BPM simulation for C2. Part one of the question posed by COMAUSATG was 'What number of personnel are required?' Although the task duration values in the BPM simulation are not statistically robust, sensitivity analysis has provided confidence to enable us to determine if more than one person is required to complete a task within a day. Using Navy's standard work day of 12 hours, if the model indicates a person will be on task for anything up to eight hours, even if the task durations are underestimated by $50 \%$ only one person would be required for the role. For cases where tasking is within four hours of the maximum, the judgement would be left to COMAUSATG.

Part two of the question posed by COMAUSATG was 'What should be the composition of the staff?' The process of collecting data to populate the model will capture this information and the answer will be designed into the model; consequently it will be a natural output of the model. 
S Tavener and P.M. Asenstorfer, Determining amphibious command and control staffing requirements using business process modelling and simulation

However, BPM is not particularly well suited to optimising the processes of a $\mathrm{C} 2$ organisation. The fact that $\mathrm{C} 2$ processes are flexible and influenced by a large number of external factors makes it extremely difficult to conduct robust comparison of alternate processes.

\section{DATA COLLECTION}

To update the 2009 BPM simulation detailed data on current practices in conducting amphibious C2 needed to be collected. This information was obtained from COMAUSATG's staff (AUSATG) during an amphibious exercise and in workshops. The exercise was a real world setting, albeit a training environment, where the staff practiced providing amphibious $\mathrm{C} 2$. The workshops were an opportunity to discuss in depth AUSATG's operating procedures.

In 2015 a small DST team attended an ADF amphibious exercise to collect data. They provided AUSATG staff specially designed booklets in which to record the tasks individuals performed during the exercise, including the start and end times of these tasks. The books included examples of the language to use and instructions on how to use them. This method was selected because detailed task information was required for the model but it was not possible to observe all staff at all times because of the size of the HQ and its use of numerous rooms on the ship. Whilst aboard, the DST staff also recorded processes they had visibility of. At the end of the exercise DST collected the booklets and manually transcribed the information provided onto a computer to enable analysis.

It was found that the booklets completed by AUSATG had varying levels of quality data. Many staff had not followed the instructions or language suggestions, which slowed the data entry and analysis process. The level of detail about tasks also varied considerably. Although there were patches of good quality data, there was insufficient to rely solely on the exercise booklets for updating the model.

To supplement the exercise data DST held workshops with AUSATG staff. AUSATG is constructed of branches, with each branch being responsible for a particular thread of an amphibious operation, such as communications, intelligence or logistics. The workshops were designed to investigate each thread separately, whilst also acknowledging the linkages between threads. The workshops usually comprised no more than five AUSATG staff and two DST personnel, one to facilitate the discussions and one as a record keeper. During these workshops the processes in the existing model were walked through and differences to current practice were noted.

The information obtained during the workshops was the most useful for updating the existing model. However, it only covered the tasks AUSATG understood they did. It did not address if that set was a complete set of what should be done. Observations of actual tasks performed would be required to validate the information obtained in the workshops. The AUSATG self-reported data from the exercises, when of good quality, was useful for verification and to provide guidance for the time taken to perform tasks.

\section{BUSINESS PROCESS MODEL SIMULATION CONSTRUCT}

Amphibious operations are conducted in phases, covering the activities from commencement and planning through to conducting actions and terminating the operation. The BPM simulation was constructed along the same lines, with one model per phase; however, for this study the focus was the planning and action phases. These phases place the greatest demands upon the AUSATG. It was assumed that if the construct of the amphibious HQ was sufficient for these phases the HQ would be capable of conducting all other phases.

The planning phase was modelled as per usual BPM, as sequential tasks and minimal branches, as demonstrated in the example in Figure 1. The end point of the planning phase model was the production and promulgation of numerous operational orders. The real time taken to perform planning was an output of simulating the model, and was influenced by the task durations within the model. The output also included, for each role, a list of tasks performed and the total time spent on tasks. 
S Tavener and P.M. Asenstorfer, Determining amphibious command and control staffing requirements using business process modelling and simulation

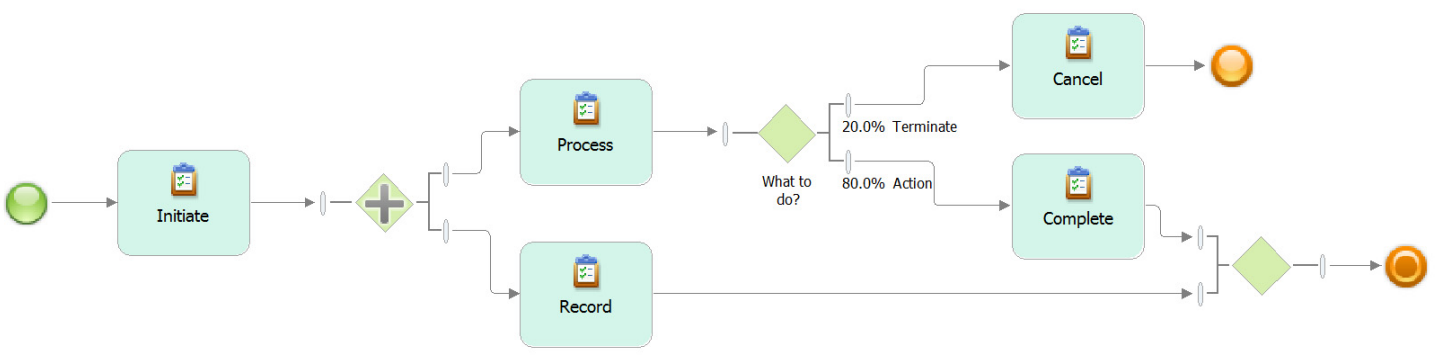

Figure 1. Example sequential BPM construct

The action phase model was constructed very differently, reflecting how an operation is conducted. When running an operation military HQ's work to a battle rhythm. The battle rhythm, captured in a document, lists against time the important meetings, inputs and outputs that are to occur each day. It is used to ensure all HQ activities are appropriately informed and key personnel are not required at more than one location at any point in time. To reflect this, many tasks in the action model were triggered by timer events rather than the previous task being completed. Figure 2 is an example of this type of model construct. Tasks were also grouped into sets covering what each branch of the amphibious HQ would undertake each day. In addition were exceptional events, such as a casualty or evacuation, that would trigger a special series of tasks that were additional to normal daily tasks.

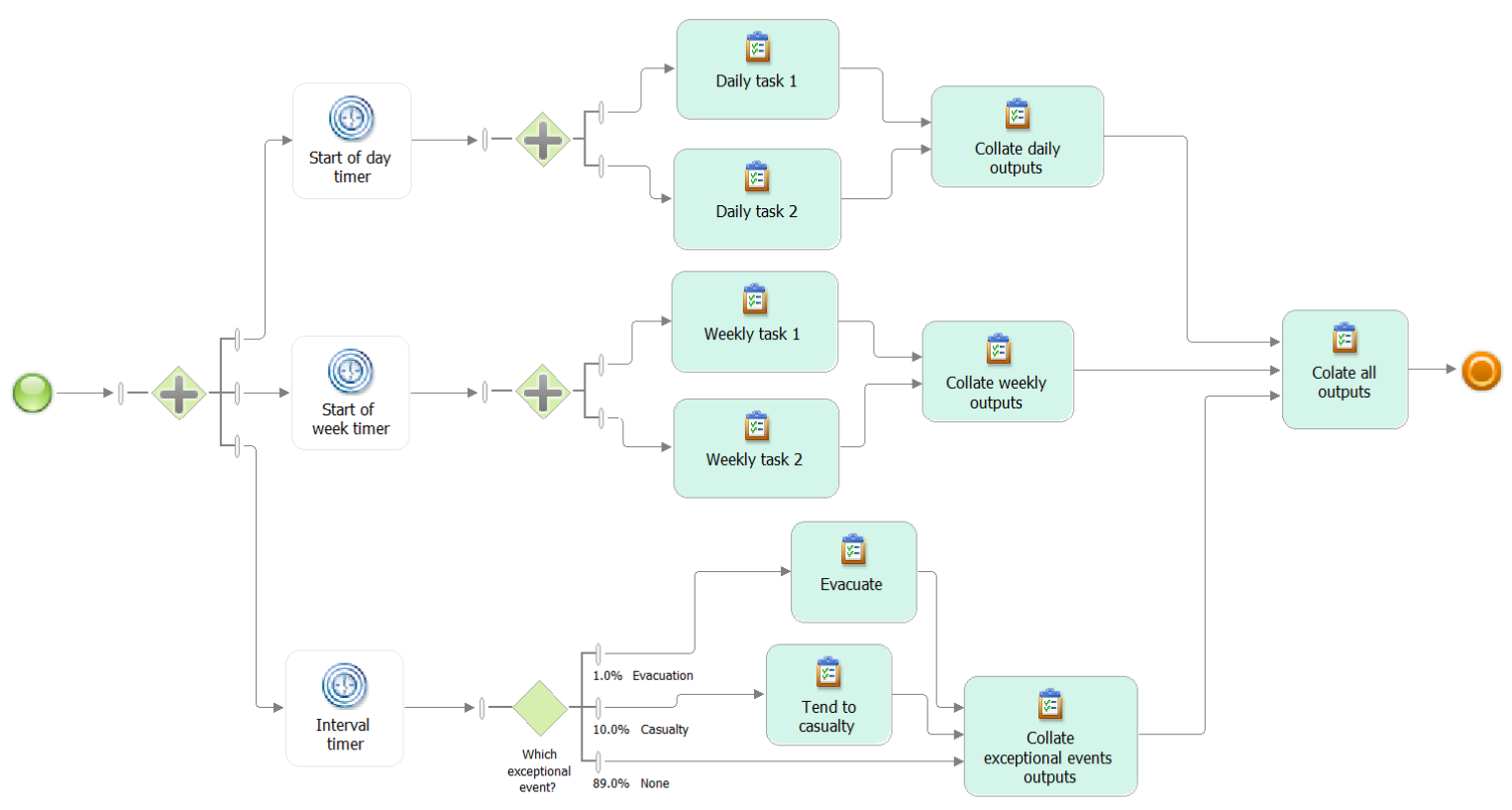

Figure 2. Example timer BPM construct

Unlike the planning model, the simulation output from the action phase was not the total time taken to undertake the action phase because this was hardwired into the model. The main output was, for each role, the list of tasks and the time spent on tasks each day. 
S Tavener and P.M. Asenstorfer, Determining amphibious command and control staffing requirements using business process modelling and simulation

The introduction of exceptional events into the simulation was via probability forks. The probability of these forks was adjusted to reflect the anticipated number of exceptional events for the type of operation being analysed.

\section{USE-CASES}

In order to estimate the different workloads generated by different mission types and scenarios, a number of representative uses cases were developed. Four mission types were selected to represent the spectrum of warfare. These four types were Humanitarian Assistance/Disaster relief (HA/DR), Non-Combatant Evacuation in a permissive environment (NEO-P), Stability and Support Operations (SASO), and high-end warfighting operations. Each of the mission types has differing rates of effort for their primary tasks and tasks which are unique for that mission type. To explore the impacts of these mission differences on the staff workloads, four separate sets of control parameters of the BPM action-phase model were developed.

The main driver for the different rates of effort for the headquarters was the number of exceptional events to which the headquarters had to respond. The headquarters has established procedures for the response to those incidents, called battle drills. For each of the four mission types, an indicative scenario was developed against which the number of incidents and hence the number and type of battle drills could be estimated. Subject matter experts (SME) then estimated incident rates for each of the battle drill types. The fork probabilities within the model were then set to reflect the estimated incident rates. For the NEO-P and SASO mission types the estimated rates of effort were within the bounds set at one end by HA/DR and at the other end by the high-end war fighting missions.

In building the use-cases it was noted that estimates for the number and type of battles drills was very sensitive to the scenario envisaged by the subject matter expert. A current project is looking to develop a framework to describe the scenarios in such a way that different SMEs will provide consistent estimates for those rates.

\section{SIMULATION DATA ANALYSIS}

The planning simulation model was run once. It was assumed all steps of planning would be performed no matter the type of mission. The type of mission would influence the time available for planning. For operations providing HA/DR there would be minimal time between being assigned the mission and conducting the operation. In contrast, high end war fighting operations would have significant notice, providing much greater time to plan.

The action simulation model was run twice, using information from the use-cases to make the differentiation. At first it was run to reflect the tempo anticipated for a HA/DR mission. Probability fork values were then adjusted to reflect an anticipated high-end war fighting tempo, and the simulation was re-run.

For all simulation runs the output was, for each role, a list of the tasks they performed and the time spent performing those tasks. During analysis, for each role, the total task duration time was converted to a proportion of the total real time as simulated in the model. This information was transformed into an interactive organisation structure diagram.

\section{INTERACTIVE ORGANISATION DIAGRAM}

An interactive colour-coded organisational structure diagram, an example of which is shown in Figure 3, was selected as the final form of the data. The aim was to provide a tool for the end user to explore different work-day constructs. 
S Tavener and P.M. Asenstorfer, Determining amphibious command and control staffing requirements using business process modelling and simulation

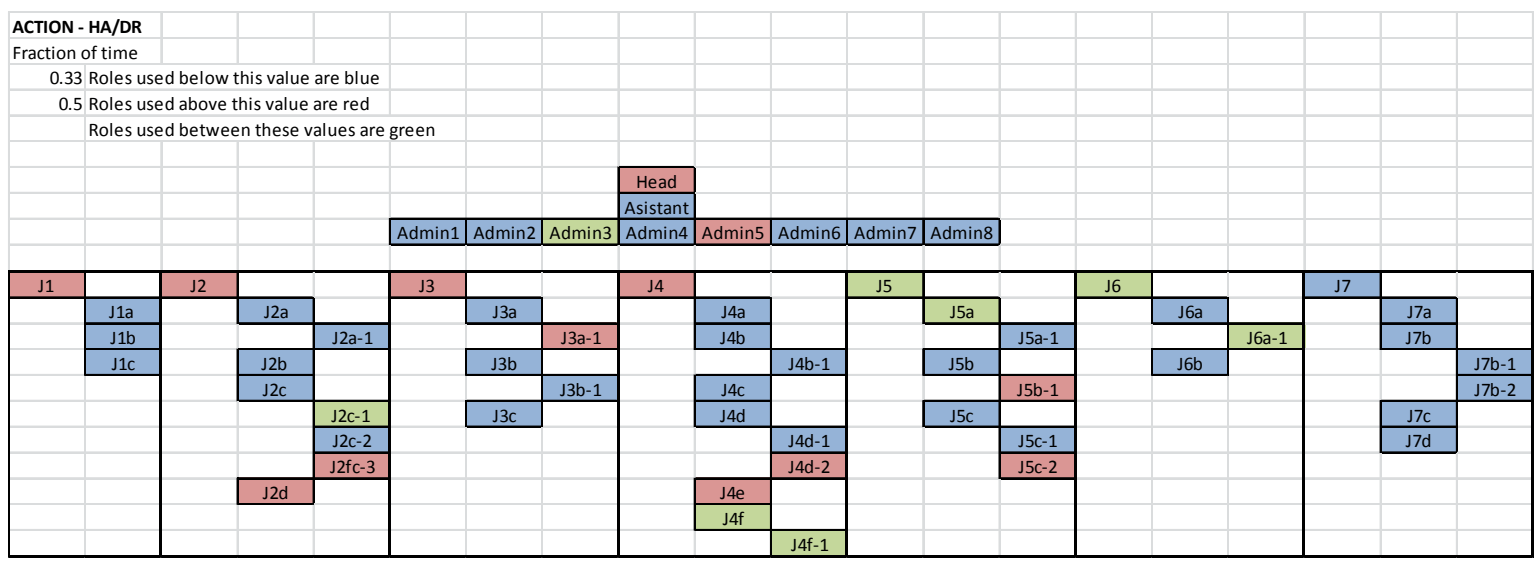

Figure 3. Example interactive organisation structure

Using an Excel (v14.0) spreadsheet the roles, as per the model, were visually placed to represent the known existing hierarchical structure. Each role was then assigned one of three colours based upon the data from the simulation model and proportion of day criteria. In the interactive spreadsheet the user specifies the lower and upper proportion of day criteria. Roles tasked with no more than the low proportion are coloured blue. Roles tasked with more than the high proportion are coloured red. Roles with time proportions between low and high are coloured green.

Adjusting the proportion of day criteria on the spreadsheet allows the user to explore different aspects of the data based upon their own definitions, such as:

- Which roles have very little tasking? (the user defines what is 'very little tasking')

- Which roles have close to a standard day of tasking?

- What changes if I change my definition of a standard day (e.g. 8 hour day to a 12 hour day)?

- Which roles are always over tasked? (the user defines what is 'over tasked')

COMAUSATG found this flexibility useful for exploring how best to structure the amphibious HQ for different types of amphibious operations. The definitions of statements such as 'very little tasking' or 'over tasked' depend upon the phase of the operation, the tempo of the operation, and criticality of actions.

\section{CONCLUSIONS AND RECOMMENDATIONS}

BPM simulation was used in this study to obtain objective data to support decisions on the appropriate construct of an amphibious C2 HQ for different types of amphibious operations. Although, it was found that BPM can be used to develop an understanding of the roles required and the anticipated workload for those roles, it is not particularly well suited to optimising the $\mathrm{C} 2$ processes used.

Data collection methods also have a significant impact upon the quality of a BPM simulation. Self-reports by personnel in an HQ during an exercise does not provide the quality of data required. Well facilitated workshops with small teams of personnel were found to be much more effective, but did require those personnel to be available outside of their normal work program. Also, direct observations during an exercise or operation would be required to validate information obtained during workshops.

Use-cases provide a common way of describing different mission types and are useful for determining appropriate values for model variables. A set of use-cases were developed for this study but further work is required to create a set that subject matter experts interpret the same way to provide consistent estimates.

The method chosen to display the model results was important in making them accessible to COMAUSATG. Flexibility to explore the impact of changing the definition of low and high workload enabled COMAUSATG to test assumptions and make a judgement on the robustness of the results.

Stemming from this study the authors would recommend:

- Confining the use of BPM simulation for $\mathrm{C} 2$ to determining the roles, tasks performed and anticipated workloads

- Use workshops for initial data collection but validate the data collected via direct observation of exercises or operations 
S Tavener and P.M. Asenstorfer, Determining amphibious command and control staffing requirements using business process modelling and simulation

- Develop a use-case framework that leads to consistent estimates from subject matter experts

- Conduct additional work to ensure the set of processes covered in the model is a complete set of all processes that should be performed. This could take the form of a functional model.

\section{ACKNOWLEDGEMENTS}

This work would not have been possible without the extensive support of our Navy client, COMAUSATG and their staff.

\section{REFERENCES}

Commonwealth of Australia (2000). Defence White Paper - Defence 2000: Our Future Defence Force, Commonwealth of Australia, Canberra.

Commonwealth of Australia (2009). Defence White Paper 2009 - Defending Australia in the Asia Pacific Century: Force 2030, Commonwealth of the Australia, Canberra. 